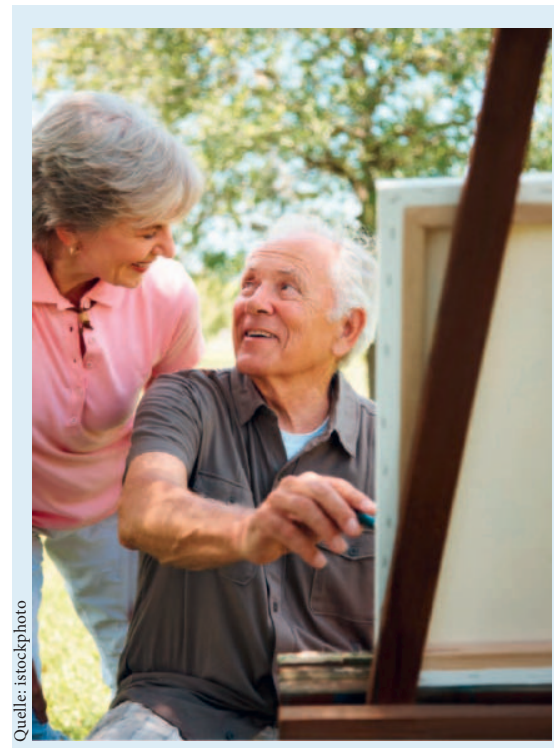

\section{Geriatrie}

Wenn sich das Leben dem Ende nähert, ist die Gesundheit besonders wichtig. Dass man Betagte motivieren kann, sich mit Tai-Chi oder Gymnastik vor Stürzen zu schützen, lesen Sie auf Seite 14. Unter psychischen Erkrankungen und dem Abbau des Intellekts leiden Betagte und ihre Familie stark. Sie lassen sich mit ganzheitlichen Verfahren behandeln, wie Sie auf dieser Seite erfahren. In unserem Expertengespräch ab Seite 9 schildert Dr. Rodolfo Mähr seine Erfahrungen beim Einsatz von Phytotherapie in einem Altersheim, während Dr. Martin Adler über den Einsatz der Naturheilkunde in einer allgemeinmedizinischen Praxis Auskunft gibt.

\title{
Ganzheitsmedizin bei psychischen Erkrankungen: Mehr Lebensqualität im Alter
}

Für neurologische Erkrankungen und psychische Probleme bieten sich verschiedene Verfahren der Komplementärmedizin an. Ihre wichtigste Wirkung ist die Erhaltung der Lebensqualität.

Viele ältere Menschen haben psychische oder neurologische Probleme. Egal, ob es sich nur um leichte Verstimmungen, um Depressionen oder um Alzheimer, Parkinson oder Demenz handelt, die Lebensqualität ist stark beeinträchtigt. Darunter leiden auch Angehörige und Pfleger. Die Nachfrage nach Methoden der Ganzheitsmedizin ist sehr gross, da viele Menschen ihnen mehr als der Schulmedizin bei der Behandlung ihrer seelischen und neurologischen Erkrankungen vertrauen. Tatsächlich können z.B. mit der Phytotherapie, der Akupunktur, der Musiktherapie oder mit Massagen Verbesserungen in der Lebensqualität erreicht werden. Daslässt sich mit einer Reihe von Metaanalysen belegen, die die Resultate vieler Studien neu auswerten.

Unter Verstimmungen und Stress leiden viele Betagte und ihr Umfeld. Es gibt Hinweise, dass etwa Multivitaminpräparate tatsächlich zu einer Aufhellung der Stimmung und zu weniger Stress bei älteren Männern führen können. Patienten, die diese Präparate nahmen, hatten weniger Depressionen und geringen Stress und waren besser in der Lage, ihren Alltag zu bewältigen. Auch Kräutermischungen und Mineralstoffe zeigten eine ähnliche Wirkung [1].

Extrakte aus Ginkgo werden in der Traditionellen Chinesischen Medizin seit langem eingesetzt und sind auch in Europa ein längst akzeptierter Teil der komplementärmedizinischen Behandlung. Anwendungsgebiete sind mentale und psychische Leiden, wie etwa Gedächtnis- oder Konzentrationsstörungen, aber auch Verwirrtheit, Depression, Angstgefühle, Niedergeschlagenheit, Tinnitus oder Kopfschmerzen. Man nimmt an, dass Ginkgoextrakte durch eine bessere Durchblutung ihre Wirkung erzielen: Die aktiven Substanzen aus dem Ginkgo sorgen für eine Erweiterung der Blutgefässe, verringern die Viskosität des Bluts und haben wahrscheinlich auch einen Einfluss auf das System der Neurotransmitter. Die Wirkstoffe sollen auch die Zahl der freien Radikale verringern.

In einer Cochrane-Studie [2] ist das Wissen über die Wirksamkeit und Verträglichkeit von Ginkgopräparaten bei Patienten zusammengetragen worden, die wegen Demenz und dem Abbau ihrer intellektuellen Leistungsfähigkeit in Behandlung waren. Die Untersuchung kommt zu dem Schluss, dass es zwischen Ginkgo und Placebo keine Unterschiede gibt, was die Häufigkeit und Schwere von Nebenwirkungen angeht. Für die Behandlungsplanung ist es wichtig, dass die Ginkgopräparate besser wirken, als es die Gabe von Placebo tut. Das trifft sowohl für unterschiedlich lange Behandlungsdauern als auch für verschiedene Dosierungen zu. Die Cochrane-Studie kommt zu dem Schluss, dass die Behandlung mit Ginkgopräparaten im Vergleich zu Placebo eine sichere Methode ist, die wenige Nebenwirkungen mit sich bringt.

Klar ist, dass Ginkgo einem Placebo z.B. bei der Verbesserung der kognitiven Fähigkeiten deutlich überlegen ist.

\section{KARGER}

Fax +497614520714 Information@Karger.d www.karger.com 
Das gilt nach 12 Wochen Behandlung bei einer Dosierung von weniger als $200 \mathrm{mg}$ pro Tag sowie bei einer Dosierung von über $200 \mathrm{mg}$ und bei allen Dosierungen nach 24 Wochen Therapiedauer. Auch bei einer Behandlungsdauer von fast einem Jahr ist Ginkgo dem Placebo überlegen, die kognitiven Fähigkeiten von älteren Erkrankten zu verbessern.

Obwohl eine grosse Zahl von Untersuchungen aus der Vergangenheit methodische Mängel aufweist, zeigen neuere Untersuchungen deutlich, dass Ginkgoextrakte eine positive Wirkung haben. Allerdings ist man noch weit davon entfernt, zu einem abschliessenden Urteil zu kommen. Die Ergebnisse von drei modernen Studien sind z.B. eher widersprüchlich, was die Grösse des Behandlungserfolgs mit Ginkgo betrifft.

Um die Selbstständigkeit älterer Menschen zu erhalten, sind tägliche Aktivitäten, die für Jüngere ganz selbstverständlich sind, sehr wichtig. Anderenfalls ist der Aufenthalt in einem Altersheim eine Konsequenz. In der Cochrane-Untersuchung haben sich die Forscher deshalb auch mit der Frage beschäftigt, ob sich mit Ginkgopräparaten auch die Fähigkeit von Älteren verbessern lässt, alleine im Alltag zurechtzukommen. Auch hier ist Ginkgo dem Placebo stets überlegen. Dies gilt sowohl für die verschiedenen Dosierungen als auch für unterschiedlich lange Behandlungsdauern. Auch bei der Therapie von Verstimmungen und Depressionen wirkt Ginkgo besser als Placebo [2].

Zudem zeigt sich auch bei Alzheimer eine Wirkung von Ginkgoextrakten. Über den Einsatz von Wirkstoffen aus Ginkgo bei dieser Erkrankung gibt es jetzt eine ganze Reihe von Untersuchungen - allerdings mit nicht einheitlichen Ergebnissen. In einer Metaanalyse [3] wurde versucht, das Material zu sichten und zu einer fundierten Aussage über die Wirksamkeit der Therapie zu gelangen. Obwohl in der Vergangenheit oft über den Einsatz von Ginkgo bei Alzheimerpatienten diskutiert wurde, konnten die Autoren nur sechs Studien in ihrer Auswertung berücksichtigen.

Während sich Unterschiede in der Grösse des Behandlungserfolgs zeigten, liess sich bei einer hohen Dosierung von über $240 \mathrm{mg}$ pro Tag stets ein positiver Effekt feststellen. Einen direkten Zusammenhang zwischen der Dosierung und dem quantitativen Behandlungserfolg konnten die Autoren allerdings nicht finden. Dafür reicht die derzeitige Datenlage nicht aus, sodass neue Studien in Angriff genommen werden müssen. Dabei wird es wichtig sein, dass die verschiedenen Untergruppen der Alzheimerpatienten jeweils einzeln betrachtet werden. Es gibt Hinweise, dass sie unterschiedlich gut auf Ginkgo ansprechen [3].

Neben der Behandlung mit Medikamenten und phytotherapeutischen Wirkstoffen spielen nichtpharmakologische Therapieansätze eine wichtige Rolle. Dabei geht es weniger darum, den Krankheitsverlauf selbst zu beeinflussen, sondern vielmehr darum, die Lebensqualität zu verbessern. Davon profitieren auch Angehörige und Pfleger, denen der Umgang mit Betroffenen, die sich wohlfühlen, leichter fällt. In einer Metaanalyse [4], die von der Stiftung Maria Wolff in Madrid gefördert wurde, konnten mehr als 1300 Studien zum Erfolg nichtpharmakologischer Therapieansätze bei Alzheimerpatienten ausgewertet werden. Knapp 180 davon waren randomisierte Studien, die den modernen Ansprüchen der klinischen Forschung genügten.

Insgesamt zeigten sich dabei sehr interessante und ermutigende Ergebnisse. Bei einer ganzen Reihe von Indikatoren konnten die verschiedenen Verfahren der Naturheilkunde Positives bewirken. Ganzheitlich behandelte Patienten mussten wesentlich später in ein Heim gehen. Bei fortgeschrittener Demenz konnte eine Verbesserung der kognitiven Leistungen erreicht werden. Die Fähigkeit, sich alleine im Alltag zurechtzufinden, liess sich auch mit den Methoden der Naturheilkunde verbessern. Insgesamt zeigte sich, dass Stimmung sowie Lebensqualität der Alzheimerpatienten davon profitierten.

Diese Ergebnisse sind auch deshalb sehr erfreulich, weil die verschiedenen Verfahren, die ohne Medikamente auskommen, kein Suchtpotenzial und weniger Nebenwirkungen haben und in der Regel auch noch preiswert sind [4].

Bei der Behandlung von Demenz setzt man häufig auf Verfahren, die nicht medikamentös sind und bei denen die Verbesserung der Lebensqualität im Zentrum steht. Dabei spielen z.B. das Wohlbefinden des Patienten, seine Gelassenheit und seine Ausgeglichenheit eine wichtige Rolle. In einer grossen Zahl von Studien konnte gezeigt werden, dass z.B. Musik oder Musiktherapie eine positive Wirkung hat. Auch Massagen und selbst Berührungen des Patienten machen sich positiv bemerkbar. Auch einfache Übungen und körperliche Aktivitäten tragen dazu bei, dass sich die Patienten besser fühlen.

Viele dieser Methoden lassen sich von Angehörigen und erst recht von professionellen Pflegerinnen und Pflegern einfach umsetzen. Sie sind in der Regel leicht zu erlernen und erfordern oft nur geringen Aufwand. Besonders Erfolg versprechend scheint zu sein, in Heimen etwa Musiktherapie oder körperliche Aktivitäten in Gruppen anzubieten. Solche Angebote sorgen für einen sozialen Austausch unter den Patienten: Sie sehen andere, können sich unterhalten, sind abgelenkt und fühlen sich deshalb wohl und gut aufgehoben [5].

Von den Patienten werden immer neue Verfahren nachgefragt, wie etwa Reiki oder unterschiedliche Formen der Berührungstherapie. Tatsächlich gibt es wissenschaftliche Evidenz, dass diese Therapien ihre Berechtigung haben: Ein Effekt lässt sich nachweisen, wenn es um die Behandlung von psychischen Beschwerden im Alter geht. In einer Metaanalyse [6], die 2010 veröffentlicht wurde, konnten fast $70 \mathrm{kli}$ nische Studien ausgewertet werden, in denen der Erfolg von Reiki oder Berüh- 
rungstherapien untersucht wurde. Dabei liessen sich signifikante Erfolge aufzeigen. Die grösste Wirkung zeigte sich bei Schmerzpatienten in der Reduktion der Schmerzintensität. Ein leichter Rückgang von negativen Verhaltenssymptomen bei dementen $\mathrm{Pa}$ tienten liess sich ebenfalls zeigen. Auch eine Auswirkung auf Angstzustände durch Berührungstherapie konnte empirisch gesichert werden.

Auch bei der Parkinsonbehandlung haben die Methoden der Ganzheitsmedizin ihren Platz. Neben der medikamentösen Therapie mit dopaminergen Substanzen gibt es eine Reihe von Möglichkeiten, Beschwerden der Patienten mit ganzheitlichen Methoden zu lindern. Eine grosse Rolle spielt dabei die Akupunktur. Jetzt zeigt sich in neuen Studien, wie die Akupunktur auf neuronaler Ebene einen Einfluss auf die Parkinsonpathologie haben könnte. Die Untersuchungen sind am Tiermodell gemacht worden, erlauben aber auch beim Menschen Rückschlüsse auf den Mechanismus. Die Akupunktur sorgt für eine Veränderung in der Ausschüttung verschiedener neuroprotektiver Substanzen - sie scheint den Spiegel zu erhöhen. In der Untersuchung [7] konnte aber auch gezeigt werden, dass nicht nur diese Substanzen vermehrt freigesetzt werden, sondern dass die Akupunktur auch einen Einfluss auf die Abbauprozesse im Nervensystem der Versuchstiere hat. Sie verlangsamt den Zelltod und reduziert den oxidativen Stress der Neurone.

Die klinische Relevanz von Akupunktur bei Parkinson lässt sich auch in Metaanalysen nachweisen. Je nach dem Design der Studie ergeben sich unterschiedliche Aussagen über die Wirksamkeit der Akupunktur. Vergleicht man z.B. in randomisierten Studien den Therapieerfolg von «richtiger» Akupunktur mit dem von Placebo, einem zufälligen Stechen von Nadeln, lässt sich kein Unterschied finden. Eine medikamentöse Behandlung in Kombination mit der Akupunktur ist wirkungsvoller als eine

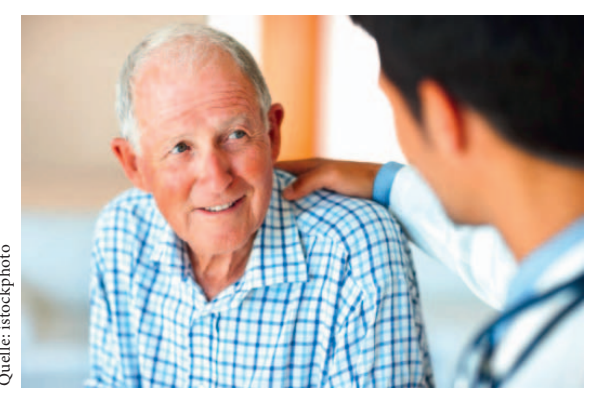

rein pharmazeutische Therapie. Wenn die Wirkung von Akupunktur mit dem Verlauf der Symptome ohne Therapie verglichen wird, zeigt sich ein leicht positiver Einfluss [8].

Diese Ergebnisse werden auch durch andere Untersuchungen bestätigt, die davon sprechen, dass es Hinweise auf eine mögliche Wirksamkeit der Akupunktur bei der Behandlung etwa der ideopathischen Parkinsonkrankheit gibt [9].

Obwohl eine Reihe von Studien vorliegt, ist es noch zu früh, die Frage abschliessend $\mathrm{zu}$ beantworten, wie wirksam die Akupunktur bei der Behandlung von Parkinson tatsächlich ist. Dafür ist die Datenbasis nicht solide genug, und es fehlen klinische Studien, die mit genügend grossen Fallzahlen arbeiten, um verlässliche Aussagen machen zu können.

Parkinson ist eine der Alterskrankheiten, bei denen Naturheilverfahren von den Patienten stark nachgefragt werden. Viele verwenden sie, um die Krankheit und ihre Symptome so erträglich wie möglich zu machen. Nach einer Untersuchung in den Vereinigten Staaten nehmen etwa zwei Drittel aller Parkinsonpatienten Multivitaminpräparate, und der Anteil der Patienten, die zu anderen Verfahren der Ganzheitsmedizin greifen, ist wahrscheinlich noch höher. Der grösste Teil dieser komplementären Therapien findet allerdings ohne Absprache mit dem Hausarzt statt. Weniger als die Hälfte der Patienten hat mit ihrem Arzt darüber gesprochen, dass sie neben den pharmakologischen Präparaten noch andere anwenden. Viele von ihnen ha- ben einen Einfluss auf die Symptome der Parkinsonkrankheit. Weiss der Hausarzt nichts von den komplementären Behandlungen, kann er schnell die falschen Schlüsse aus möglichen Veränderungen des Patienten ziehen. Das betrifft nicht nur die motorischen Symptome der Parkinsonkrankheit, sondern auch andere Symptome, wie etwa Störungen der Atmung, der Verdauung und des Schlafs oder eine gedrückte Stimmung. Für Ärzte, die Parkinsonpatienten behandeln, ist es deshalb wichtig, dass die Komplementärmedizin auf die medizinische Behandlung mit dopaminergen Substanzen abgestimmt ist [10]. (rfi)

\section{Literatur}

1 Harris E, Kirk J, Rowsell R, Vitetta L, Sali A, Scholey AB, Pipingas A: The effect of multivitamin supplementation on mood and stress in healthy older men. Hum Psychopharmacol 2011;26:560-567.

2 Birks J, Grimley EV, Van Dongen M: Ginkgo biloba for cognitive impairment and dementia. Cochrane Database Syst Rev 2002;4:CD003120. Update in Cochrane Database Syst Rev 2007; 2:CD003120.

3 Janssen IM, Sturtz S, Skipka G, Zentner A, Velasco Garrido M, Busse R: Ginkgo biloba in Alzheimer's disease: a systematic review. Wien Med Wochenschr 2010;160:539-546.

4 Olazarán J, Reisberg B, Clare L et al: Nonpharmacological therapies in Alzheimer's disease: a systematic review of efficacy. Dement Geriatr Cogn Disord 2010;30:161-178.

5 Hulme C, Wright J, Crocker T, Oluboyede Y, House A: Non-pharmacological approaches for dementia that informal carers might try or access: a systematic review. Int J Geriatr Psychiatry 2010;25:756-763.

6 Jain S, Mills PJ: Biofield therapies: helpful or full of hype? A best evidence synthesis. Int J Behav Med 2010;17:1-16.

7 Joh TH, Park HJ, Kim SN, Lee H: Recent development of acupuncture on Parkinson's disease. Neurol Res 2010;32(suppl 1):5-9.

$>$ Lee MS, Shin BC, Kong JC, Ernst E: Effectiveness of acupuncture for Parkinson's disease: a systematic review. Mov Disord 2008;23:1505-1515.

9 Lam YC, Kum WF, Durairajan SS, Lu JH, Man SC, Xu M, Zhang XF, Huang XZ, Li M: Efficacy and safety of acupuncture for idiopathic Parkinson's disease: a systematic review. J Altern Complement Med 2008;14:663-671.

10 Zesiewicz TA, Evatt ML: Potential influences of complementary therapy on motor and nonmotor complications in Parkinson's disease. CNS Drugs 2009;23:817-835. 